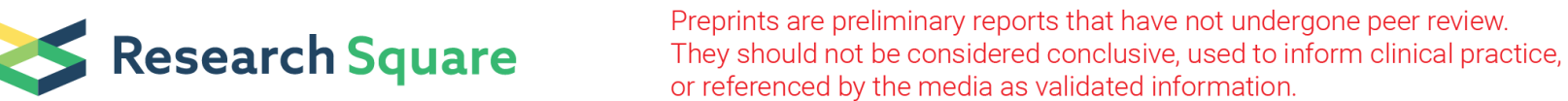

\section{Casein phosphopeptide acts synergistically with fluoride to inhibit initial adhesion of Streptococcus mutans to the saliva-coated hydroxyapatite disc}

\section{Xiaodie Wang}

Sun Yat-Sen University

Limin Liu

Sun Yat-Sen University

\section{Xiaoyan Zhou}

The University of Sydney

\section{Yongbiao Huo}

Sun Yat-Sen University

Jinlong Gao ( $\nabla$ jinlong.gao@sydney.edu.au )

The University of Sydney

Haijing Gu ( $\nabla$ guhj@mail.sysu.edu.cn )

Sun Yat-Sen University

\section{Research article}

Keywords: Casein phosphopeptide, Fluoride, Bacterial adhesion, Salivary pellicle, Dental caries, Streptococci mutans

Posted Date: March 10th, 2020

DOl: https://doi.org/10.21203/rs.3.rs-16587/v1

License: (c) (i) This work is licensed under a Creative Commons Attribution 4.0 International License.

Read Full License

Version of Record: A version of this preprint was published at BMC Oral Health on June 12th, 2020. See the published version at https://doi.org/10.1186/s12903-020-01158-8. 


\section{Abstract}

Background Recent preventive strategies for dental caries focus on targeting the mechanisms underlying biofilm formation, including inhibiting bacterial adhesion. A promising approach to prevent bacterial adhesion is to modify the composition of acquired salivary pellicle. This in vitro study investigated the effect and possible underlying mechanism of pellicle modification by casein phosphopeptide (CPP) on Streptococcus mutans (S. mutans) initial adhesion, and the impact of fluoride on the efficacy of CPP.

Methods The salivary pellicle-coated hydroxyapatite (s-HA) disc was modified by $2.5 \%$ CPP or $2.5 \%$ CPP supplemented with $900 \mathrm{ppm}$ fluoride solutions. After cultivation of S. mutans, the adherent bacteria were visualized by scanning electron microscopy (SEM) and quantitatively evaluated using the plate count method. Confocal laser scanning microscopy (CLSM) was used to evaluate the proportions of total and dead S. mutans. The concentrations of total, free, and bound calcium and fluoride in CPP and fluoridedoped CPP solutions were determined. The water contact angle and zeta potential of s-HA with and without modification were measured. The data were statistically analyzed using one-way ANOVA followed by a Turkey post hoc multiple comparison test.

Results Initial adhesion of S. mutans to s-HA was inhibited in response to pellicle modification by CPP and fluoride-doped CPP, and the latter was more efficient. CLSM analysis showed that the proportion of dead S. mutans did not differ between the groups. Water contact angle and zeta potential decreased after pellicle modification, and both were lowest in the fluoride-doped CPP group.

Conclusions Pellicle modification by CPP inhibited S. mutans initial adhesion to s-HA, possibly by reducing hydrophobicity and negative charge on the s-HA surface, and incorporating fluoride into CPP enhanced its anti-adhesion effect.

\section{Background}

Dental caries is a multi-factor lifestyle-related disease caused by the effect of metabolic end products of dental biofilm on the tooth surface [1]. A dental biofilm is a community of microorganisms that are embedded in their extracellular polymeric substances [2]. Biofilm formation is initiated by bacterial adhesion to the acquired salivary pellicle on the surface of dental hard tissues [3]. Many microorganisms participate in the development of caries. Streptococcus mutans (S. mutans) is considered the primary etiologic agent because of its excellent acidogenicity, aciduricity and glucan synthesis ability [4]. Pellicle formation starts immediately after toothbrushing through the selective adsorption of salivary components onto the tooth surface. The pellicle has a protective effect against dental caries by functioning as a semi-permeable barrier and a calcium and phosphate reservoir to inhibit demineralization and promote remineralization [5]. However, specific receptors in the pellicle, such as amylase and proline-rich proteins, can be recognized and bound by lectin-like bacterial adhesins, thereby facilitating bacterial adhesion [6]. Conventional treatment of biofilm-related diseases aims at mechanically removing the biofilms. However, recent therapeutic strategies focus on targeting the 
mechanisms underlying biofilm formation, including inhibiting bacterial adhesion [7]. Bacterial adhesion can be disrupted when the molecular composition and physicochemical properties of the pellicle are altered [8]. Modification of the pellicle by plant extracts or ingredients of natural products, such as tannic acid and propolis, is a safe and cost-effective approach to prevent bacterial adhesion $[9,10]$.

Casein phosphopeptide (CPP) are a subgroup of milk-derived phosphorylated peptides that can be naturally present in milk or obtained by proteolytic digestion of casein [11-13]. CPP contains the cluster sequence $-\operatorname{Ser}(\mathrm{P})-\operatorname{Ser}(\mathrm{P})$-Ser $(\mathrm{P})$-Glu-Glu-, and this highly polar acidic domain has high binding affinity for divalent metal ions [14]. CPP can efficiently bind and solubilize calcium, thereby enhancing the passive absorption of calcium in the gastrointestinal tract, improving remineralization of the tooth surface, and buffering the $\mathrm{pH}$ of dental biofilm [15]. The inhibitory effect of CPP on dental enamel demineralization has been confirmed in vitro and in vivo $[16,17]$. A randomized controlled trial showed that toothpaste containing $2 \% \mathrm{CPP}$ or $1190 \mathrm{mg} / \mathrm{kg}$ fluoride similarily reduced the incidence of caries [18]. The cariespreventive activity of CPP is attributed to its ability to stabilize high levels of amorphous calcium phosphate (ACP) on the tooth surface [19], and the CPP-amorphous calcium phosphate (CPP-ACP) complex has been developed and patented by the Reynolds group [20]. However, limited information is available on the effect of CPP on bacterial activity. $0.5 \%$ CPP solution had no significant effect of on the established S. mutans biofilm [21]. A previous study indicated that CPP inhibited S. mutans and Streptococcus sobrinus (S. sobrinus) initial adhesion to the pellicle-coated hydroxyapatite surface [22]. However, the exact anti-adhesion mechanism of CPP remains unknown.

Fluoride has been recognized as an anti-caries agent for more than seven decades; it can inhibit demineralization and promote remineralization of dental hard tissues [23]. It also affects bacterial viability by acting as a glycolytic enzyme inhibitor as well as a transmembrane proton carrier, which leads to cytoplasmic acidification [24]. It has been proposed that, incorporating fluoride as a phosphatase inhibitor into the CPP-containing dental care products can enhance the effectiveness of CPP during application [25]. Although several studies showed that incorporating fluoride into CPP-ACP improves the remineralization [26, 27], there is also evidence indicating that fluoride supplementation did not improve the demineralization-suppressing effect of CPP or CPP-ACP $[16,19,28,29]$. The impact of fluoride on the efficacy of CPP remains unclear.

The aim of this study was to investigate the effect and possible underlying mechanism of CPP on pellicle modification and S. mutans initial adhesion, as well as the impact of fluoride on the efficacy of CPP.

\section{Methods}

\section{Casein phosphopeptide and fluoride}

CPP powder (Fujifilm Wako Pure Chemical Corporation, Osaka, Japan) and sodium fluoride (Sigma, Saint Louis, MO, USA) used in this study were reagent-grade.

\section{Test solutions}


Two-fold serially diluted CPP solutions in the range of $0.15625-10 \%(\mathrm{w} / \mathrm{v})$ and $2.5 \% \mathrm{CPP}$ solution supplemented with $900 \mathrm{ppm}$ fluoride were prepared. The $\mathrm{pH}$ of solutions was adjusted to 7.0 using $\mathrm{NaOH}$, and the solutions were sterilized through a $0.22 \mu \mathrm{m}$ polyethersulfone membrane (Merck Millipore Ltd., Tullagreen, Carrigtwohill, Co. Cork, IRL). Heat-inactivated 2.5\% CPP solution was prepared by autoclaving at $121^{\circ} \mathrm{C}$ for $15 \mathrm{~min}$. All solutions were prepared freshly and used on the same day.

\section{Hydroxyapatite disc}

Hydroxyapatite (HA) disc $(9.7 \mathrm{~mm}$ in diameter and $1.5 \mathrm{~mm}$ in thickness, Clarkson Chromatography Products, South Williamsport, PA, USA) was used as the substrate in this study. To visualize the live and dead bacteria cells on the substrate surface, the HA disc was polished using a precision lapping/polishing machine (Unipol-1502, Kejing Auto-Instrument Co., LTD, Shenyang, China) with \#360, \#600, and \#1000 silicon carbide sandpapers under constant water cooling, until the disc reached a thickness of $0.3 \mathrm{~mm}$. After polishing, the disc was sonicated in ultrapure water for $10 \mathrm{~min}$ to remove the debris. Before experiments, the HA disc was autoclaved at $121^{\circ} \mathrm{C}$ for $15 \mathrm{~min}$.

\section{Bacterial strain and culture conditions}

S. mutans UA159 (ATCC 700610) purchased from Guangdong Microbial Culture Collection Center was used in this study. To prepare the inoculum, S. mutans was firstly recovered on a brain heart infusion (BHI, Difco, Detroit, MI, USA) agar plate supplemented with $5 \%$ sterile defibrinated sheep blood for $48 \mathrm{~h}$ at $37^{\circ} \mathrm{C}$ under anaerobic conditions $\left(5 \% \mathrm{CO}_{2}, 10 \% \mathrm{H}_{2}, 85 \% \mathrm{~N}_{2}\right)$. A single colony was selected and inoculated into $10 \mathrm{~mL}$ of $\mathrm{BHI}$ broth and incubated anaerobically at $37^{\circ} \mathrm{C}$ overnight. Bacteria was harvested by centrifugation (3000 rpm, $4^{\circ} \mathrm{C}, 5 \mathrm{~min}$ ), washed twice with sterile phosphate buffered saline (PBS), and finally re-suspended in $\mathrm{BHI}$ broth. The optical density at $600_{\mathrm{nm}}\left(\mathrm{OD}_{600 \mathrm{~nm}}\right)$ was adjusted to 0.2 (corresponding to a concentration of approximately $2.0 \times 10^{8} \mathrm{cell} / \mathrm{sL}$ ).

\section{Saliva collection and preparation}

Human whole unstimulated saliva was collected from healthy volunteers with informed consent, and the procedure was approved by the Ethical Review Committee of Sun Yat-Sen University (No. ERC-[2017]-24). Saliva samples were collected and prepared according to a published method with some modification [30]. Briefly, saliva was collected from eight volunteers who had not taken in anything but water for at least $2 \mathrm{~h}$ before saliva collection, were never smokers, and had not consumed any antibiotics or mouthwashes for at least 3 months. The saliva was spitted into a sterile and iced centrifuge tube until 10 $\mathrm{mL}$ of saliva was obtained, and this process usually took about 50-60 minutes. The saliva from eight donors was pooled. After centrifugation at $4000 \mathrm{rpm}$ and $4{ }^{\circ} \mathrm{C}$ for $20 \mathrm{~min}$, the supernatant was collected and filtered using a $0.22 \mu \mathrm{m}$ polyethersulfone membrane. Clarified saliva samples were frozen quickly in liquid nitrogen, and stored at $-80^{\circ} \mathrm{C}$. Saliva collection from the same donors was carried out with an interval of at least 30 days, until the present study was completed. Saliva samples were used within 6 months and thawed at room temperature prior to experiments. 
The HA discs were individually placed into a 24-well plate (Costar, Corning, NY, USA). A volume of $1 \mathrm{~mL}$ of the clarified saliva was added into each well and incubated at $37^{\circ} \mathrm{C}$ for $2 \mathrm{~h}$. After rinsing twice with sterile PBS to obtain salivary pellicle-coated HA (s-HA) discs, the discs were randomly divided into eight groups ( $n=6$ per group): control group (PBS), and seven treated groups containing serially diluted concentrations of CPP. The s-HA disc was incubated in $1 \mathrm{~mL}$ of the corresponding solutions for $2 \mathrm{~h}$ at 37 ${ }^{\circ} \mathrm{C}$, followed by two rinses with PBS. A volume of $1 \mathrm{~mL}$ of the bacteria suspension was seeded and incubated for 30 min and $2 \mathrm{~h}$ in a humidified atmosphere of $5 \% \mathrm{CO}_{2}$ at $37^{\circ} \mathrm{C}$ (three discs per group and incubation time). After rinsing twice with PBS to remove non-attached and loosely bound bacteria, the HA disc was transferred into an eppendorf tube containing $2 \mathrm{~mL}$ of PBS. Adherent $S$. mutans was detached by sonication for $10 \mathrm{~min}$, followed by vortexing for a further 60 seconds. The sonicated and vortexed $S$. mutans suspension was serially diluted and $100 \mu \mathrm{L}$ of the diluted solution was spread over a $\mathrm{BHI}$ agar plate and incubated for $48 \mathrm{~h}$ at $37^{\circ} \mathrm{C}$ in a humidified atmosphere of $5 \% \mathrm{CO}_{2}$. The number of adherent $S$. mutans was expressed as colony-forming units (CFU) per disc. The experiment was repeated three times in triplicate. The adhesion reduction percentage was calculated as follows: (CFU counts of the control group-CFU counts of the treated group)/ CFU counts of the control group $\times 100 \%$.

\section{Bacterial adhesion assay}

$\mathrm{CPP}$ at $2.5 \%(\mathrm{w} / \mathrm{v})$ was used for further experiments based on the ability to reduce the adhesion of $S$. mutans by approximately $50 \%$ [31]. For the $S$. mutans adherence assay, s-HA disc was prepared as described above. The s-HA discs were randomly divided into four groups ( $n=10$ per group): (1) blank control (PBS); (2) negative control (heat-inactivated 2.5\% CPP); (3) CPP (2.5\% CPP); and (4) CPP + F (2.5\% CPP supplemented with $900 \mathrm{ppm}$ fluoride). Pellicle modification and inoculation of $S$. mutans were performed as described above (three discs per group and incubation time). The number of adherent $S$. mutans was expressed as CFU per disc. The experiment was repeated three times in triplicate.

Scanning electron microscopy (SEM) was used to visualize S. mutans adhesion on the HA surface. Two samples from each group and incubation time were prefixed in $2.5 \%$ glutaraldehyde at room temperature for at least $3 \mathrm{~h}$. After washing four to six times using ultrapure water, samples were dehydrated using gradient concentrations of ethanol $(30 \%, 50 \%, 70 \%, 80 \%, 85 \%, 90 \%, 95 \%$, and $100 \%)$ for $15 \mathrm{~min}$, and then substituted by tert butyl alcohol three times, freeze-dried, sputter-coated with gold, and examined by SEM (JSM-6330F, JEOL, Japan).

\section{Bacteria LIVE/DEAD staining}

Adhesion of S. mutans was also measured using a LIVE/DEAD BackLight Bacterial Viability Kit (L7012, Thermo Scientific, USA). The polished HA discs with a thickness of $0.3 \mathrm{~mm}$ were used. Pellicle formation and modification as well as cultivation of $S$. mutans were performed as described above (three HA discs per group and incubation time). After incubation for $30 \mathrm{~min}$ and $2 \mathrm{~h}$, the HA disc was washed twice with $0.9 \% \mathrm{NaCl}$ and then stained with $1 \mathrm{~mL}$ of LIVE/DEAD ${ }^{\circledR}$ BacLight $^{\text {TM }}$ solution at room temperature in the 
dark for $15 \mathrm{~min}$. A volume of $1 \mathrm{~mL}$ of LIVE/DEAD ${ }^{\circledR}$ BacLight $^{\text {TM }}$ solution contained $997 \mu \mathrm{L}$ of ultrapure water, $1.5 \mu \mathrm{L}$ of propidium iodide (PI), and $1.5 \mu \mathrm{L}$ of SYTO 9, which was prepared according to the manufacturer's instructions. Dead bacterial cells with damaged membrane were finally stained in red color by PI, while live bacterial cells with intact cell membrane were stained in green color by SYTO 9. Samples were observed by a confocal laser scanning microscopy (CLSM, LSM 780, Zeiss, Oberkochen, BW, Germany) with a 20x water-immersion objective lens. Dual-channel scanning observations were performed through a green channel for SYTO 9 (excitation wavelength: $488 \mathrm{~nm}$ ) and a red channel for PI (excitation wavelength: $543 \mathrm{~nm}$ ). Four fields were randomly selected on each sample for scanning. Image analysis was performed with ImageJ software. The ratio of the area occupied by green or red fluorescence to the whole area on each visual field was measured. For each specimen, the ratio (\%) of the area covered by total fluorescence of each color to the whole area was calculated. Subsequently, the proportion (\%) of red to total fluorescence was measured. The experiment was replicated three times.

\section{Ion quantified analysis}

The total and free calcium and fluoride concentrations in 2.5\% CPP and 2.5\% CPP supplemented with 900 ppm fluoride solutions were quantified, and the corresponding bound ion concentrations were calculated. Before detecting the total ion concentrations, $1 \mathrm{~mL}$ of the initial solution was diluted with 19 $\mathrm{mL}$ of $1.0 \mathrm{M} \mathrm{HNO}_{3}$ and reacted for $24 \mathrm{~h}$, followed by centrifugation at $1000 \mathrm{~g}$ for $15 \mathrm{~min}$ at room temperature; the supernatant was collected to detect the total ion concentration [32]. Before detection of free ion concentrations, macromolecular CPP with a molecular weight of approximately $3 \mathrm{kDa}$ was filtered using a magnetically stirred ultrafiltration device (Amicon, Model 8200, $200 \mathrm{~mL}$, Millipore) equipped with an ultrafiltration disc (1 kDa NMWL, PLAC06210, Ultracel ${ }^{\circledR}$ regenerated cellulose, Millipore). According to the instructions, $50 \mathrm{~mL}$ of the initial solution was added into the device; equipped with a magnetic stirrer at $300 \mathrm{rpm}$, the ultrafiltration separation was driven using high-purity $\mathrm{N}_{2}(99.999 \%)$ and the pressure was maintained within $2.4 \mathrm{~atm}$. CPP was filtered and the filtrate was collected to detect the free ion concentrations. Fluoride concentration was detected by ion chromatography (IC-1100, Thermo Fisher Scientific, USA), and calcium concentration was measured using inductively coupled plasmaoptical emission spectroscopy (ICP-OES, ICP-OES 730, Agilent, USA). Calcium and fluoride concentrations were expressed as $\mathrm{mmol} / \mathrm{L}(\mathrm{mM})$.

\section{Hydrophobicity determination}

The hydrophobicity of the s-HA surface with and without modification was determined by measuring the water contact angle with a Contact Angle Meter (DMo-501, Kyowa Kogyo CO., LTD, Japan). Deionized water was used as the medium ( $2 \mu \mathrm{L}$ per drop), and the sessile drop technique was employed. The right and left water contact angles for each droplet were measured at room temperature and averaged. For each sample, the measurement was repeated at three randomly selected regions. Finally, the result was expressed as degree $\left(^{\circ}\right)$.

\section{Zeta potential analysis}


The electrophoretic mobilities of pellicle coated ceramic hydroxyapatite powder (Clarkson Chromatography Products, Williamsport, PA, USA) with and without modification were measured by a Zeta Potential Analyzer (Zetasizer Nano ZS90; Malvern Instruments, Malvern, UK) according to a published method [33]. The ceramic hydroxyapatite powder is the raw material for the HA disc that was used in the current study. Both the ceramic hydroxyapatite powder and HA disc were purchased from the same company. Pellicle formation and modification were carried out as described above in an incubator shaker (50 rpm) at $37^{\circ} \mathrm{C}$. Five measurements of electrophoretic mobilities for each sample were averaged, and the zeta potential was calculated according to the Helmholtz-Smoluchowski formula.

\section{Statistical analysis}

For all statistical analyses, SPSS v.19.0 software (IBM, Armonk, NY, USA) was used. All values were expressed as the mean \pm standard deviation (SD). The inter-group differences were estimated by one-way analysis of variance (ANOVA) followed by a Turkey post hoc multiple comparison test. The level of significance was set at $p<0.05$.

\section{Results}

\section{Effect of serially diluted CPP on S. mutans initial adhesion}

As shown in Fig. 1, CPP inhibited the initial adhesion of $S$. mutans in a dose-dependent manner. At 30 min of incubation, compared with the control group, the reduction in the adhesion of $S$. mutans was $12.6 \%$, $36.8 \%, 49.6 \%, 76.2 \%$, and $82.9 \%$ for CPP at concentrations of $0.63 \%$ to $10 \%$, successively and respectively ( $0.63 \%$ CPP: $p<0.01$; others: $p<0.0001$ ). At $2 \mathrm{~h}$ of incubation, the adhesion of $S$. mutans was reduced by $32.9 \%, 51.5 \%, 78.5 \%$, and $83.8 \%$ for CPP at concentrations of $1.25 \%$ to $10 \%$, successively and respectively (all: $p<0.0001$ ). Although the adhesion reduction in the $0.63 \% \mathrm{CPP}$ group was $8.9 \%$ at $2 \mathrm{~h}$ of incubation, the difference was not significant $(p=0.094)$. There were no significant differences between $0.16 \% \mathrm{CPP}, 0.31 \% \mathrm{CPP}$, and the control group at $30 \mathrm{~min}$ or $2 \mathrm{~h}$.

\section{Effect of CPP and fluoride-doped CPP on S. mutans initial adhesion to s-HA}

The effect of 2.5\% CPP and 2.5\% CPP supplemented with $900 \mathrm{ppm}$ fluoride on the initial adhesion of $S$. mutans to s-HA was visualized by SEM (Fig. 2a) and quantitatively evaluated by the plate count method (Fig. 2b).

SEM images showed the distribution of $S$. mutans on HA discs with different coatings. The images showed a lower distribution of $S$. mutans in the CPP and CPP + F groups than that in the control groups, both at $30 \mathrm{~min}$ and $2 \mathrm{~h}$, and the CPP $+\mathrm{F}$ group showed the lowest distribution. Quantitative analysis using the plate count method showed no significant difference between the blank control and the negative control at $30 \mathrm{~min}(p=0.409)$ and $2 \mathrm{~h}(p=0.877)$. At $30 \mathrm{~min}$ of incubation, the CFU counts were $50.8 \%$ and $74.0 \%$ lower in the CPP and CPP + F groups, respectively, than in the blank control (both: $p<$ $0.0001)$. The amount of $S$. mutans in the CPP $+F$ group was reduced by $47.2 \%$ compared with that in the 
CPP group $(p<0.0001)$. Similar results were obtained at $2 \mathrm{~h}$ of incubation: the CFU counts were $48.4 \%$ and $74.5 \%$ lower in the CPP and CPP + F groups, respectively, than in the blank control (both: $p<0.0001$ ), and $50.6 \%$ lower in the CPP $+\mathrm{F}$ group than in the CPP group $(p<0.0001)$.

\section{Live and dead analysis}

Live and dead analyses were performed to confirm the anti-adhesion effect of CPP and fluoride-doped $\mathrm{CPP}$, and to estimate their antimicrobial properties. As shown in Fig. 3a, fluorescence was lower in the $\mathrm{CPP}$ and $\mathrm{CPP}+\mathrm{F}$ groups than that in the controls both at $30 \mathrm{~min}$ and $2 \mathrm{~h}$. The ratio of the area occupied by total fluorescence to the whole area for the blank control, negative control, CPP, and CPP + F groups was $11.19 \%, 11.17 \%, 5.43 \%$, and $2.73 \%$ at 30 min of incubation, and $20.00 \%, 19.84 \%, 9.71 \%$, and $4.59 \%$ at $2 \mathrm{~h}$ of incubation, respectively (Fig. $3 \mathrm{~b}$ ). The differences between groups were consistent with the results of the plate count method. The area (\%) occupied by S. mutans in the CPP and CPP + F groups was $51.43 \%$ and $75.64 \%$ lower at $30 \mathrm{~min}$ and was $51.47 \%$ and $77.06 \%$ lower at $2 \mathrm{~h}$ than that in the blank control, respectively (all: $p<0.0001$ ). The area occupied by $S$. mutans in the CPP + F group was $49.84 \%$ lower at $30 \mathrm{~min}$ and $52.43 \%$ lower at $2 \mathrm{~h}$ than that in the CPP group (both: $p<0.05$ ). There was no significant difference between the blank and negative controls at $30 \mathrm{~min}(p=0.873)$ or $2 \mathrm{~h}(p=0.714)$.

The proportion (\%) of red fluorescence (dead S.mutans) to total fluorescence was $2.08 \%, 2.23 \%, 2.47 \%$, and $2.59 \%$ at $30 \mathrm{~min}$, and $1.61 \%, 1.95 \%, 2.27 \%$, and $2.43 \%$ at $2 \mathrm{~h}$, for the blank control, negative control, $\mathrm{CPP}$, and CPP + F groups, respectively (Fig. 3c). There were no differences between the control (blank and negative controls) and experimental groups (CPP and CPP $+\mathrm{F}$ groups) (30 $\min : p=0.061 ; 2 \mathrm{~h}: p=0.213)$.

\section{Calcium and fluoride concentrations in experimental solutions}

The concentrations of total, free and bound calcium and fluoride in $2.5 \%$ CPP and $2.5 \%$ CPP supplemented with $900 \mathrm{ppm}$ fluoride solutions are shown in Table 1. The $2.5 \% \mathrm{CPP}$ solution $(\mathrm{pH} 7.0)$ contained $30.00 \mathrm{mM} \mathrm{Ca}^{2+}$, but did not contain $\mathrm{F}^{-}$, and $29.08 \mathrm{mM}(96.9 \%) \mathrm{Ca}^{2+}$ was bound by CPP. The 2.5\% CPP solution supplemented with 900 ppm fluoride contained $47.35 \mathrm{mM}(899.65 \mathrm{ppm}) \mathrm{F}^{-}$, and 33.75 $\mathrm{mM}(641.25 \mathrm{ppm}, 71.3 \%) \mathrm{F}^{-}$was bound by CPP, and the other was similar to the $2.5 \%$ CPP solution.

Table 1 Concentrations of total, free, and bound calcium and fluoride in the experimental solutions.

\begin{tabular}{|c|c|c|c|c|c|c|}
\hline & \multicolumn{6}{|c|}{ Ion concentration $\square \mathrm{mM} \square$} \\
\hline & \multicolumn{2}{|c|}{ Total } & \multicolumn{2}{|l|}{ Free } & \multicolumn{2}{|l|}{ Bound } \\
\hline & $\mathrm{Ca}$ & $\mathrm{F}$ & $\mathrm{Ca}$ & $\mathrm{F}$ & $\mathrm{Ca}$ & $\mathrm{F}$ \\
\hline $\mathrm{CPP}$ & $30.00 \pm 0.12$ & - & $0.92 \pm 0.03$ & - & $29.08(96.9 \%)$ & - \\
\hline $\mathrm{CPP}+\mathrm{F}$ & $30.41 \pm 0.04$ & $47.35 \pm 0.13$ & $0.78 \pm 0.03$ & $13.60 \pm 0.15$ & 29.63 (97.4\%) & $33.75(71.3 \%)$ \\
\hline
\end{tabular}

CPP: 2.5\% CPP solution; CPP + F: 2.5\% CPP supplemented with 900 ppm fluoride solution; Ca: Calcium; F: Fluoride. The concentrations of total and free calcium and fluoride in solutions were measured, and the corresponding bound ion concentrations were calculated. Data are expressed as the mean \pm SD. Percent of ion bound by CPP is indicated in parentheses. 


\section{Water contact angle measurement}

The hydrophobicity of the s-HA surface with and without modification was estimated by measuring the water contact angle. Representative images are shown in Fig. 4, and the results are shown in Table 2. Water contact angle significantly decreased from $42.0^{\circ}$ (blank control) to $24.1^{\circ}(\mathrm{CPP})$ and $17.4^{\circ}(\mathrm{CPP}+\mathrm{F})$ (both: $p<0.0001)$, and was smaller in the CPP $+\mathrm{F}$ group than in the CPP group $(p<0.05)$. The water contact angle was higher in the negative control group $\left(55.9^{\circ}\right)$ than in the other groups (all: $\left.p<0.0001\right)$.

Table 2 Water contact angle and zeta potential of the HA with different coatings.

\begin{tabular}{lll}
\hline & Water Contact Angle (degree) & Zeta Potential $(\mathrm{mV})$ \\
\hline Blank control & $42.0 \pm 1.1^{\mathrm{a}}$ & $-9.8 \pm 0.2^{\mathrm{a}}$ \\
Negative control & $55.9 \pm 3.4^{\mathrm{b}}$ & $-11.5 \pm 0.7^{\mathrm{b}}$ \\
\hline $\mathrm{CPP}$ & $24.1 \pm 0.8^{\mathrm{c}}$ & $-16.4 \pm 0.3^{\mathrm{c}}$ \\
\hline $\mathrm{CPP}+\mathrm{F}$ & $17.4 \pm 0.8^{\mathrm{d}}$ & $-19.4 \pm 0.3^{\mathrm{d}}$ \\
\hline$p$-value & $<0.0001$ & $<0.0001$ \\
\hline
\end{tabular}

Blank control: salivary pellicle-coated HA; Negative control, CPP and CPP + F: salivary pellicle-coated HA modified by heat-inactivated 2.5\% CPP, 2.5\% CPP, and 2.5\% CPP supplemented with $900 \mathrm{ppm}$ fluoride. Data are expressed as the mean \pm SD. Within columns (water contact angle or zeta potential), different superscript letters $(a, b, c, d)$ indicated significant difference $(p<0.05)$.

\section{Zeta potential analysis}

All s-HA surfaces with or without modification exhibited negative zeta potential values, and the results are shown in Table 2. The zeta potential value for the blank control was $-9.8 \mathrm{mV}$, which decreased to -11.5 , -16.4, and -19.4 mV for the negative control, CPP, and CPP + F groups, respectively (all: $p<0.0001$ ). The value for the CPP $+\mathrm{F}$ group was significantly lower than that for the CPP group $(p<0.0001)$.

\section{Discussion}

A promising approach to prevent dental caries is to reduce the abundance of S. mutans in dental biofilm by interfering with its initial adhesion [34,35]. Pellicle modification is recognized as an alternative option for improving the anti-adhesion properties of the tooth surface [36]. The present study provides evidence of the anti-adhesion effect of CPP and fluoride-doped CPP on S. mutans. In addition, this is the first study to show that the net negative charge and hydrophilicity of the s-HA surface increase after pellicle modification by CPP, and fluoride further enhances the efficacy of CPP.

An in vitro model was established to simulate pellicle formation and modification as well as initial adhesion of S. mutans. We did not perform experiments on natural enamel because individual enamel specimens had different chemical compositions and unclear previous history [23]. Hence, the HA disc, 
which is the major component of enamel, was used as the model substrate in the present study. Our results demonstrated that CPP inhibited the initial adhesion of S. mutans in a dose-dependent manner; a concentration of $2.5 \%$ CPP reduced the adhesion of S. mutans by approximately $50 \%$, and this CPP concentration was selected for the current study [31]. Fluoride is commonly added to various oral care products. To investigate the effect of fluoride on the efficacy of CPP, a commonly used concentration of fluoride (900 ppm) was selected for incorporation into CPP [23].

In the present study, the effect of pellicle modification by CPP and fluoride-doped CPP on S. mutans initial adhesion was evaluated using the plate count method, SEM and live-dead staining. And the incubation times of $30 \mathrm{~min}$ and $2 \mathrm{~h}$ were chosen to better understand the nonspecific and specific interactions between S. mutans and the substrate [8]. The results showed an apparent reduction of adherent $\mathrm{S}$. mutans on the modified s-HA surface. The inhibitory effect was most obvious in the fluoride-doped CPPmodified pellicle group. Heat-inactivated CPP was used as the negative control to confirm the effect of CPP in the active form. There was no difference between the blank control (non-modified pellicle) and negative control groups, indicating that denaturing CPP eliminated the anti-adhesion activity. As shown in the live-dead staining analysis, the proportion of red fluorescence (dead S. mutans) to the total fluorescence was similar among the four groups. This suggested that the anti-adhesion effect of CPP and fluoride-doped CPP was non-bactericidal, namely S. mutans was prevented from attaching to the modified s-HA surface rather than being killed after adhesion. This non-bactericidal therapeutic approach targeting at bacterial adhesion can avoid the selective pressure on microorganisms, which may induce resistance to treatment or proliferation of opportunistic pathogens [37].

Calcium is commonly used as an aggregation-inducing agent during the production of CPP [25]. In the present study, we performed ion quantification to measure the free and bound calcium and fluoride concentrations in the CPP and fluoride-doped CPP solutions. The results showed that the $2.5 \% \mathrm{CPP}$ solution contained $30 \mathrm{mM}$ calcium. Moreover, $29 \mathrm{mM}$ calcium and $641 \mathrm{ppm}$ fluoride were bound by CPP in the 2.5\% CPP supplemented with 900 ppm fluoride solution. This suggests that CPP can bind and stabilize calcium and fluoride to prevent the formation of $\mathrm{CaF}_{2}$ precipitates.

CPP adsorption and bacterial adhesion to the pellicle are both driven by specific and non-specific interactions, such as van der Waals, electrostatic, and hydrophobic interactions [3, 38]. To investigate the effect of pellicle modification on surface properties, the zeta potential and water contact angle were measured to evaluate the charge and hydrophobicity of the s-HA surface before and after pellicle modification. Surface charge and hydrophobicity of the substrate are two important determinants that influence bacterial adhesion [39]. Decreased surface hydrophobicity is correlated with decreased bacterial adhesion [40], because initial bacterial adhesion involves the interaction between hydrophobic components on the surface of bacteria and those on the substrate [41]. Increasing the net negative charge of the tooth surface reduces Streptococcal adhesion [42], because Streptococcal species (e.g., S. mutans) typically have a negatively charged surface [43]. The present study showed that both zeta potential and water contact angle decreased after pellicle modification by CPP. This suggested that the formation of a more hydrophilic and negatively charged surface increased the repulsion between S. 
mutans and the substrate. CPP is an amphiphiles and negatively charged peptide $[25,44]$. An alternative mechanism mediating adherence of CPP to the pellicle is the localization of hydrophobic sites to the pellicle surface and the orientation of hydrophilic sites toward the outer environment [15]. As shown in the present study, CPP increases the hydrophilicity of the s-HA surface. A previous study suggested that the calcium maximally bound by CPP is adequate to neutralize the intrinsic negative charge of CPP [44]. Although $29 \mathrm{mM}$ calcium was bound by CPP in this study, the zeta potential of s-HA decreased after pellicle modification by CPP. We assumed that the amount of calcium bound by CPP was not saturated, or calcium was partially released after CPP absorption onto the pellicle, or other anions bound by CPP partially eliminated the effect of calcium. This is the first study demonstrating that CPP can increase the hydrophilicity and negative charge of the s-HA surface, and this synergistic effect partially led to the reduction of S. mutans initial adhesion. A recent study indicated that CPP selectively binds to certain pellicle components, including salivary acidic proline-rich phosphoprotein 1/2 precursor and mucin 7 [38], which are also binding sites for S. mutans [45]. Therefore, it is reasonable to speculate that the number of binding sites for S.mutans decreased on the CPP-modified pellicle, which decreased the affinity of S.mutans for the CPP-modified s-HA surface. Although the caries-preventive activity of CPP is known to be mediated by its ability to stabilize high concentrations of ACP on the tooth surface, the anti-adhesion activity of CPP demonstrated in the present study may be partially responsible for its caries-preventive effect.

Our results showed that the anti-adhesion effect was most efficient in the fluoride-doped CPP-modified pellicle group, which showed the lowest water contact angle and zeta potential values. Fluoride inhibits the adhesion of Streptococcus sanguis to the s-HA surface even at low concentrations [46]. The considerable amount of free fluoride (258.4 ppm) in the fluoride-doped CPP solution may play a role in inhibiting S. mutans adhesion by further decreasing the negative charge and hydrophobicity of the s-HA surface $[23,47]$. The bound fluoride $(641 \mathrm{ppm})$ may have played a role in the present study as well. On one hand, fluoride may have increased the net negative charge of CPP, and fluoride-doped CPP modified the s-HA surface. On the other hand, fluoride may be partially released because of degradation of CPP by peptidases and/or phosphatases from S. mutans [48], and/or interaction between CPP and specific pellicle components [38]. The gradually released fluoride could continually affect the s-HA surface. Although the antimicrobial property of fluoride against cariogenic bacteria has been documented [24], the anti-adhesion effect of fluoride-doped CPP on S. mutans was non-bactericidal, as demonstrated in this study. The results suggested that the bound fluoride molecules did not act on bacteria directly. Free fluoride may have been incorporated onto the pellicle only during the pellicle modifying process and was washed away or diluted during subsequent steps. In general, the bound and free fluoride might function synergistically to enhance the anti-adhesion effect of CPP.

The present study is limited in that a single bacterial strain was investigated, and the impact of other strains was not assessed. The effects on a greater number of bacterial species should be assessed in future studies. 


\section{Conclusion}

The results of this study demonstrated that initial adhesion of S. mutans to S-HA can be effectively inhibited by pellicle modification by CPP, and incorporating fluoride into CPP increases its efficacy. We propose, for the first time, that the anti-adhesion effect of CPP and fluoride-doped CPP is non-bactericidal, and that it is mediated, at least in part, by their effect on the non-specific interactions (electrostatic and hydrophobic interactions) between S. mutans and s-HA. The present study provides new insight into the caries-preventive mechanism of CPP. Combination of CPP, as an anti-caries agent, with fluoride may improve its effect.

\section{Abbreviations}

CPP: Casein phosphopeptides; S. mutans: Streptococcus mutans; S. sobrinus: Streptococcus sobrinus; CPP-ACP: Casein phosphopeptides-amorphous calcium phosphate; BHI: Brain heart infusion; PBS:

Phosphate buffered saline; HA: Hydroxyapatite; s-HA: Salivary pellicle-coated hydroxyapatite; CFU: Colony forming units; SEM: Scanning electron microscopy; CLSM: Confocal laser scanning microscopy; PI: Propidium iodide; SD: Standard deviation (SD)

\section{Declarations}

\section{Ethics approval and consent to participate}

Ethics approval for saliva collection was obtained from the Ethical Review Committee, Guanghua School of Stomatology, Sun Yat-sen University (Approval No. ERC-[2017]-24), and written informed consent was obtained from the donors.

\section{Consent for publication}

Not applicable.

\section{Availability of data and materials}

The datasets used and/or analyzed during the current study available from the corresponding author on reasonable request.

\section{Competing interests}

The authors declare that they have no competing interest.

\section{Funding}

This work was supported by grants fromOpen Fund of Guangdong Provincial Key Laboratory of Oral Diseases, Sun Yat-Sen University (grant number KF2017120102), and Youth Program of National Natural Science Foundation of China (grant number 81700983). 


\section{Authors' contributions}

$\mathrm{HG}$, and JG designed the study. XW performed the experiments and collected the data. $\mathrm{LM}, \mathrm{XZ}$ and $\mathrm{YH}$ analysed the data. XW and LM wrote the manuscript. XZ, YH, JG, and HG revised the manuscript. All authors have read and approved the final manuscript.

\section{Acknowledgements}

Not applicable.

\section{References}

1. Chu J, Zhang T, He K. Cariogenicity features of Streptococcus mutans in presence of rubusoside. BMC Oral Health. 2016;16:54.

2. Kim AR, Ahn KB, Kim HY, Seo HS, Yun CH, Han SH. Serine-rich Repeat Adhesin Gordonii Surface Protein B is Important for Streptococcus gordonii Biofilm Formation. J Endod. 2016;42:1767-72.

3. Hannig M, Joiner A. The structure, function and properties of the acquired pellicle. Monogr Oral Sci. 2006;19:29-64.

4. Loesche WJ. Role of Streptococcus mutans in human dental decay. Microbiol Rev. 1986;50:353-80.

5. Hannig M, Hannig C. The pellicle and erosion. Monogr Oral Sci. 2014;25:206-14.

6. Douglas CW. Bacterial-protein interactions in the oral cavity. Adv Dent Res. 1994;8:254-62.

7. Klemm P, Vejborg RM, Hancock V. Prevention of bacterial adhesion. Appl Microbiol Biotechnol. 2010;88:451-9.

8. Cheaib Z, Rakmathulina E, Lussi A, Eick S. Impact of Acquired Pellicle Modification on Adhesion of Early Colonizers. Caries Res. 2015;49:626-32.

9. Hertel S, Potschke S, Basche S, Delius J, Hoth-Hannig W, Hannig M, Hannig C. Effect of Tannic Acid on the Protective Properties of the in situ Formed Pellicle. Caries Res. 2017;51:34-45.

10. Habluetzel A, Schmid C, Carvalho TS, Lussi A, Eick S. Impact of honey on dental erosion and adhesion of early bacterial colonizers. Sci Rep. 2018;8:10936.

11. Meisel H, Bernard H, Fairweather-Tait S, FitzGerald RJ, Hartmann R, Lane CN, McDonagh D, Teucher $\mathrm{B}, \mathrm{Wal} \mathrm{JM}$. Detection of caseinophosphopeptides in the distal ileostomy fluid of human subjects. $\mathrm{Br}$ J Nutr. 2003;89:351-9.

12. Baum F, Ebner J, Pischetsrieder M. Identification of multiphosphorylated peptides in milk. J Agric Food Chem. 2013;61:9110-7.

13. Dallas DC, Citerne F, Tian T, Silva VL, Kalanetra KM, Frese SA, Robinson RC, Mills DA, Barile D. Peptidomic analysis reveals proteolytic activity of kefir microorganisms on bovine milk proteins. Food Chem. 2016;197:273-84.

14. Reynolds EC. The prevention of sub-surface demineralization of bovine enamel and change in plaque composition by casein in an intra-oral model. J Dent Res. 1987;66:1120-7. 
15. Nongonierma AB, Fitzgerald RJ. Biofunctional properties of caseinophosphopeptides in the oral cavity. Caries Res. 2012;46:234-67.

16. White AJ, Gracia LH, Barbour ME. Inhibition of dental erosion by casein and casein-derived proteins. Caries Res. 2011;45:13-20.

17. Ferrazzano GF, Cantile T, Quarto M, Ingenito A, Chianese L, Addeo F. Protective effect of yogurt extract on dental enamel demineralization in vitro. Aust Dent J. 2008;53:314-9.

18. Rao SK, Bhat GS, Aradhya S, Devi A, Bhat M. Study of the efficacy of toothpaste containing casein phosphopeptide in the prevention of dental caries: a randomized controlled trial in 12- to 15-year-old high caries risk children in Bangalore, India. Caries Res. 2009;43:430-5.

19. Rechmann P, Bekmezian S, Rechmann BMT, Chaffee BW, Featherstone JDB. MI Varnish and MI Paste Plus in a caries prevention and remineralization study: a randomized controlled trial. Clin Oral Investig. 2018;22:2229-39.

20. Reynolds EC. Anticariogenic complexes of amorphous calcium phosphate stabilized by casein phosphopeptides: a review. Spec Care Dentist. 1998;18:8-16.

21. Dashper SG, Catmull DV, Liu SW, Myroforidis H, Zalizniak I, Palamara JE, Huq NL, Reynolds EC. Casein Phosphopeptide-Amorphous Calcium Phosphate Reduces Streptococcus mutans Biofilm Development on Glass lonomer Cement and Disrupts Established Biofilms. PLoS One. 2016;11:e0162322.

22. Schupbach P, Neeser JR, Golliard M, Rouvet M, Guggenheim B. Incorporation of caseinoglycomacropeptide and caseinophosphopeptide into the salivary pellicle inhibits adherence of mutans streptococci. J Dent Res. 1996;75:1779-88.

23. Loskill P, Zeitz C, Grandthyll S, Thewes N, Muller F, Bischoff M, Herrmann M, Jacobs K. Reduced adhesion of oral bacteria on hydroxyapatite by fluoride treatment. Langmuir. 2013;29:5528-33.

24. Nassar HM, Gregory RL. Biofilm sensitivity of seven Streptococcus mutans strains to different fluoride levels. J Oral Microbiol. 2017;9:1328265.

25. FitzGerald RJ. Potential Uses of Caseinophosphopeptides. Int Dairy J. 1998;8:451-7.

26. Sinfiteli PP, Coutinho TCL, Oliveira PRA, Vasques WF, Azevedo LM, Pereira AMB, Tostes MA. Effect of fluoride dentifrice and casein phosphopeptide-amorphous calcium phosphate cream with and without fluoride in preventing enamel demineralization in a $\mathrm{pH}$ cyclic study. J Appl Oral Sci. 2017;25:604-11.

27. Hamba H, Nikaido T, Inoue G, Sadr A, Tagami J. Effects of CPP-ACP with sodium fluoride on inhibition of bovine enamel demineralization: a quantitative assessment using micro-computed tomography. J Dent. 2011;39:405-13.

28. Poggio C, Gulino C, Mirando M, Colombo M, Pietrocola G. Preventive effects of different protective agents on dentin erosion: An in vitro investigation. J Clin Exp Dent. 2017;9:e7-e12.

29. Wiegand A, Attin T. Randomised in situ trial on the effect of milk and CPP-ACP on dental erosion. J Dent. 2014;42:1210-5. 
30. Jakubovics NS, Robinson JC, Samarian DS, Kolderman E, Yassin SA, Bettampadi D, Bashton M, Rickard AH. Critical roles of arginine in growth and biofilm development by Streptococcus gordonii. Mol Microbiol. 2015;97:281-300.

31. Li BC, Li XL, Lin HC, Zhou Y. Curcumin as a Promising Antibacterial Agent: Effects on Metabolism and Biofilm Formation in S. mutans. Biomed Research International. 2018.

32. Fernando JR, Shen P, Sim CPC, Chen YY, Walker GD, Yuan Y, Reynolds C, Stanton DP, MacRae CM, Reynolds EC. Self-assembly of dental surface nanofilaments and remineralisation by SnF2 and CPPACP nanocomplexes. Sci Rep. 2019;9:1285.

33. Bezerra SJC, Joao-Souza SH, Aoki IV, Borges AB, Hara AT, Scaramucci T. Anti-Erosive Effect of Solutions Containing Sodium Fluoride, Stannous Chloride, and Selected Film-Forming Polymers. Caries Res. 2019;53:305-13.

34. Gibbons RJ. Adherent interactions which may affect microbial ecology in the mouth. J Dent Res. 1984;63:378-85.

35. Marine J, Myers CP, Picquet GA, Zaidel LA, Wu D, Uhrich KE. Reduction of bacterial attachment on hydroxyapatite surfaces: Using hydrophobicity and chemical functionality to enhance surface retention and prevent attachment. Colloids Surf B Biointerfaces. 2018;167:531-7.

36. Campoccia D, Montanaro L, Arciola CR. A review of the biomaterials technologies for infectionresistant surfaces. Biomaterials. 2013;34:8533-54.

37. Davies J, Davies D. Origins and evolution of antibiotic resistance. Microbiol Mol Biol Rev. 2010;74:417-33.

38. Huq NL, Myroforidis H, Cross KJ, Stanton DP, Veith PD, Ward BR, Reynolds EC. The Interactions of CPP-ACP with Saliva. Int J Mol Sci. 2016;17.

39. Song F, Koo H, Ren D. Effects of Material Properties on Bacterial Adhesion and Biofilm Formation. J Dent Res. 2015;94:1027-34.

40. Yang X, Huang P, Wang H, Cai S, Liao Y, Mo Z, Xu X, Ding C, Zhao C, Li J. Antibacterial and antibiofouling coating on hydroxyapatite surface based on peptide-modified tannic acid. Colloids Surf $B$ Biointerfaces. 2017;160:136-43.

41. Colloca ME, Ahumada MC, Lopez ME, Nader-Macias ME. Surface properties of lactobacilli isolated from healthy subjects. Oral Dis. 2000;6:227-33.

42. Reynolds EC, Wong A. Effect of adsorbed protein on hydroxyapatite zeta potential and Streptococcus mutans adherence. Infect Immun. 1983;39:1285-90.

43. Roger V, Tenovuo J, Lenander-Lumikari M, Soderling E, Vilja P. Lysozyme and lactoperoxidase inhibit the adherence of Streptococcus mutans NCTC 10449 (serotype c) to saliva-treated hydroxyapatite in vitro. Caries Res. 1994;28:421-8.

44. Cross KJ, Huq NL, Palamara JE, Perich JW, Reynolds EC. Physicochemical characterization of casein phosphopeptide-amorphous calcium phosphate nanocomplexes. J Biol Chem. 2005;280:15362-9. 
45. Gibbons RJ, Cohen L, Hay DI. Strains of Streptococcus mutans and Streptococcus sobrinus attach to different pellicle receptors. Infect Immun. 1986;52:555-61.

46. Eifert R, Rosan B, Golub E. Optimization of an hydroxyapatite adhesion assay for Streptococcus sanguis. Infect Immun. 1984;44:287-91.

47. de Jong HP, de Boer P, van Pelt AW, Busscher HJ, Arends J. Effect of topically applied fluoride solutions on the surface free energy of pellicle-covered human enamel. Caries Res. 1984;18:505-8.

48. Reynolds EC, Riley PF. Protein dissimilation by human salivary-sediment bacteria. J Dent Res. 1989;68:124-9.

\section{Figures}
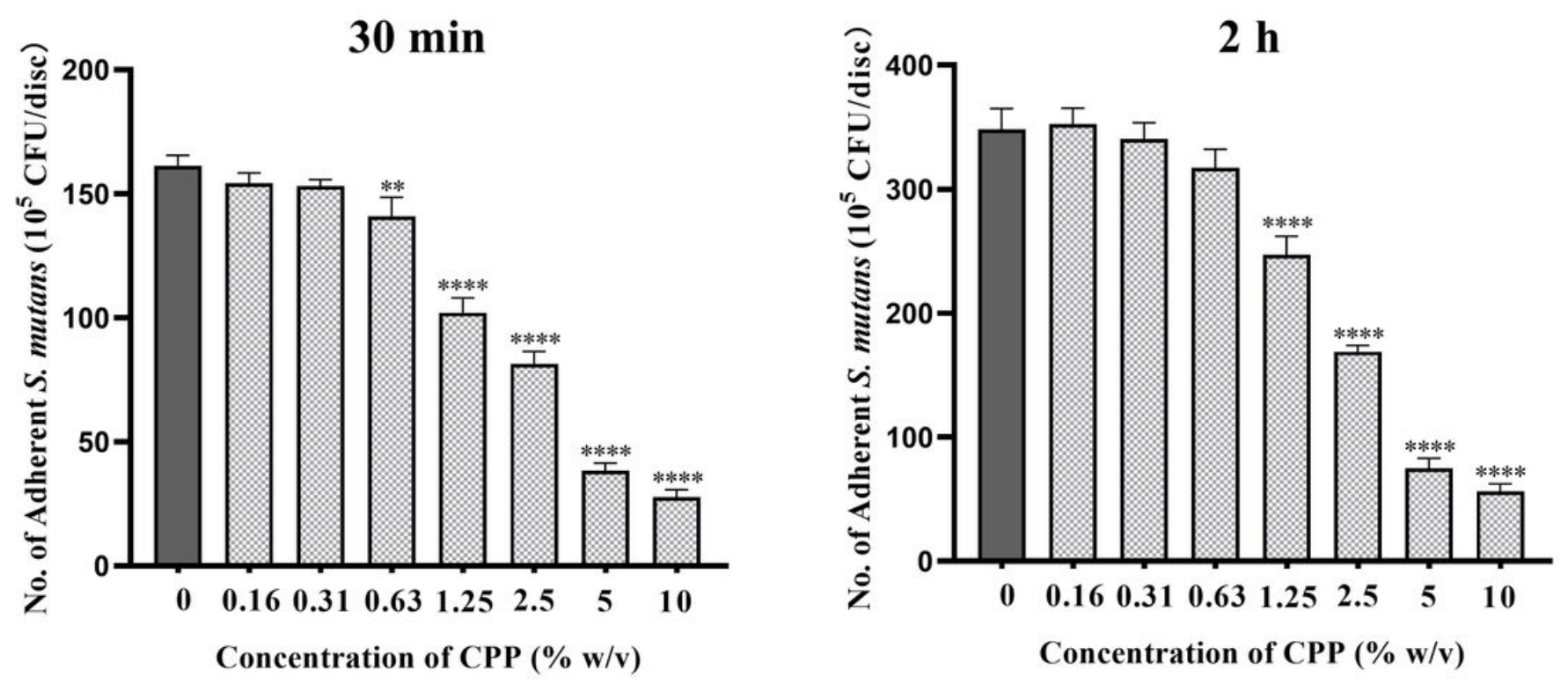

Figure 1

Effects of different concentrations of CPP on S. mutans initial adhesion. Salivary pellicle-coated HA disc was modified by two-fold serially diluted CPP solutions in the range of $0.15625-10 \%$, followed by cultivation of $\mathrm{S}$. mutans for $30 \mathrm{~min}$ and $2 \mathrm{~h}$. Colony forming units (CFU) of S. mutans per disc were determined and the results were expressed as the mean \pm SD. ${ }^{* *} p<0.01$, $* \star \star * p<0.0001$ compared with control. 

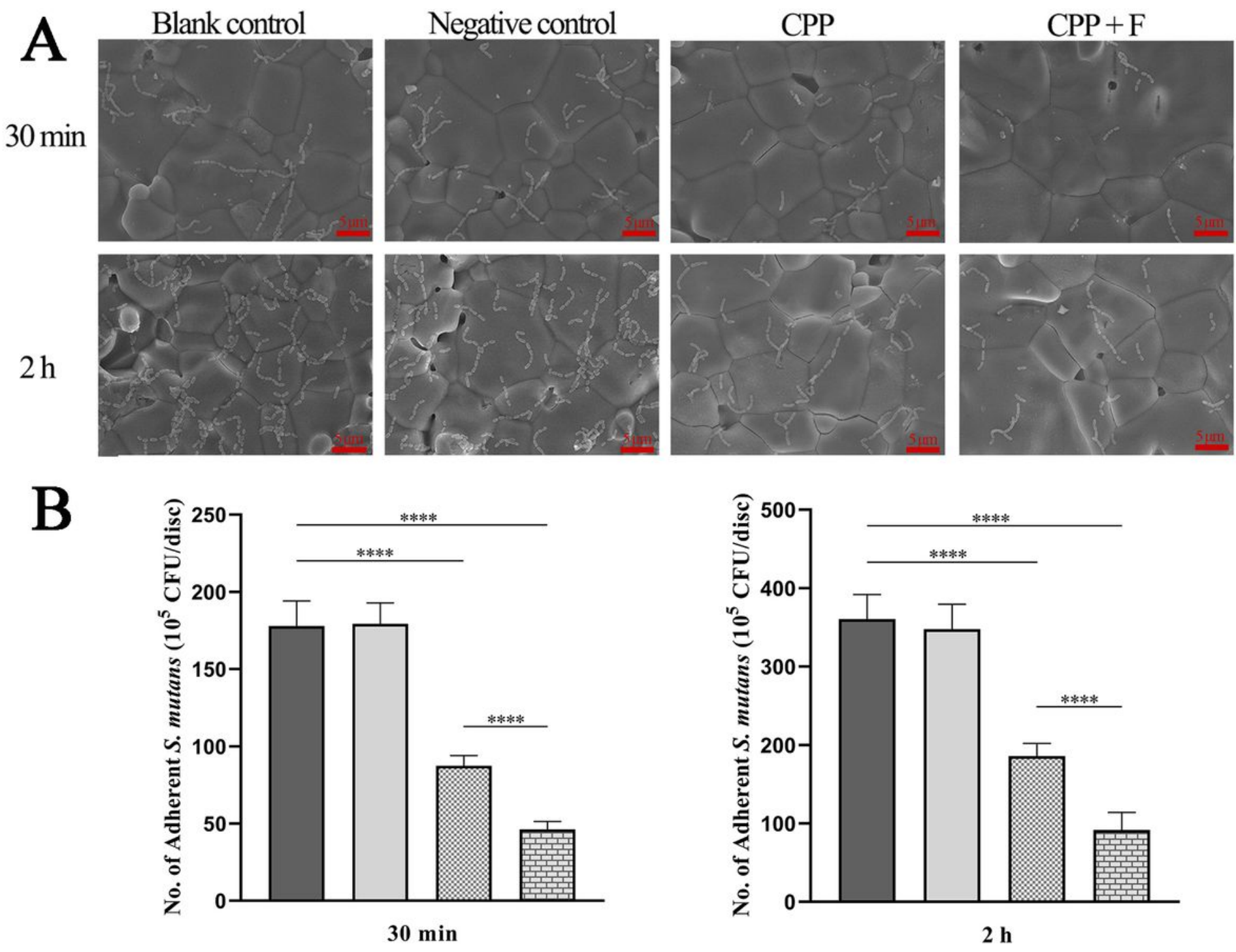

Blank Control $\square$ Negative Control $\square$ CPP 回 CPP+F

Figure 2

Effect of pellicle modification by CPP and fluoride-doped CPP on S. mutans initial adhesion. HA discs were divided into four groups: salivary pellicle-coated HA (blank control), salivary pellicle-coated HA modified by heat-inactivated 2.5\% CPP (negative control), or 2.5\% CPP (CPP), or $2.5 \%$ CPP supplemented with 900 ppm fluoride (CPP + F). (a) Representative SEM images (3000-fold magnification) at 30 min and $2 \mathrm{~h}$ of incubation. (b) Colony-forming unit (CFU) counts of $\mathrm{S}$. mutans per disc was calculated according

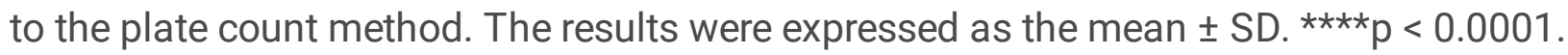



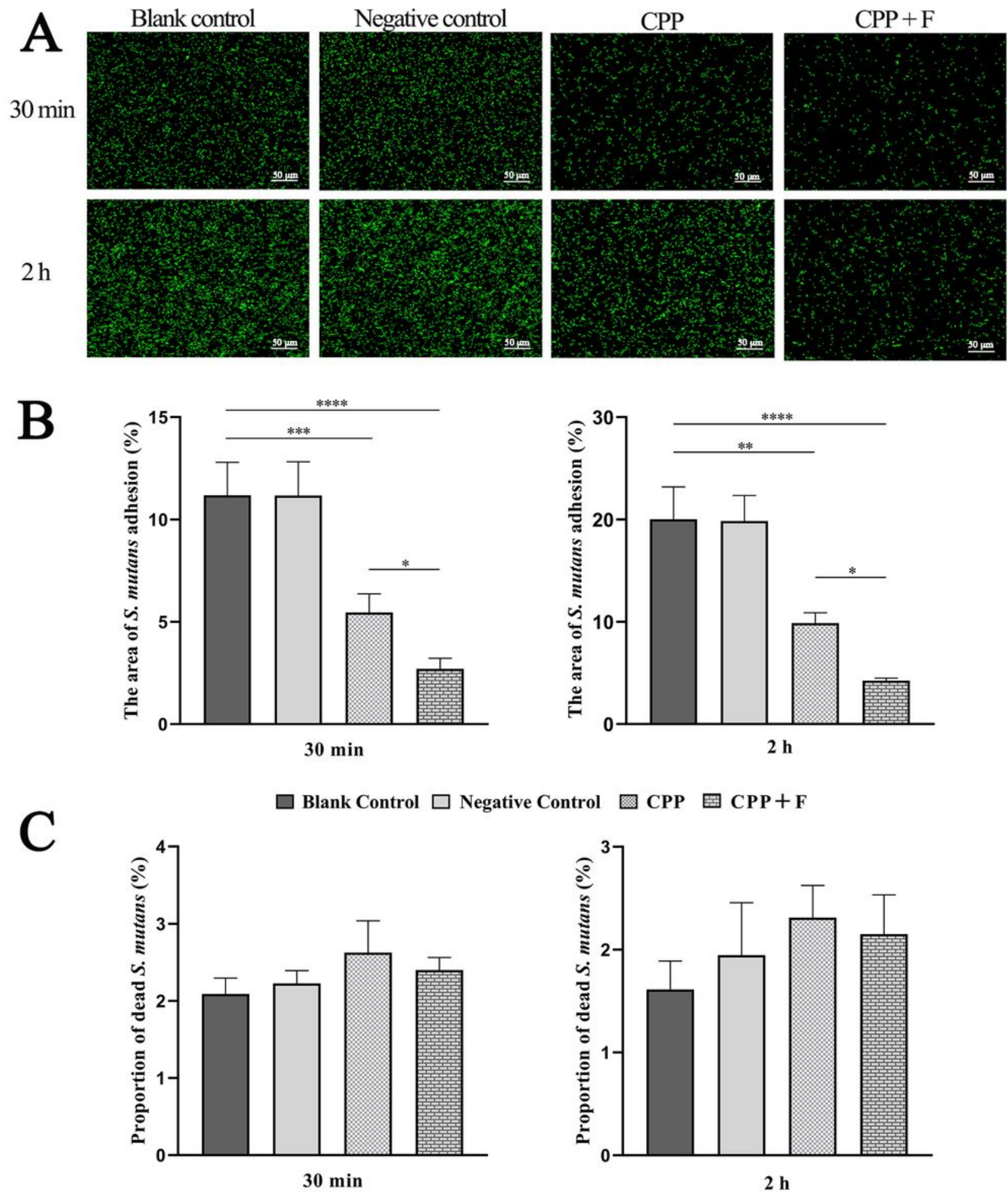

Figure 3

Live/dead staining analysis of S. mutans initial adhesion to HA discs with different coatings. HA discs were divided into four groups: salivary pellicle-coated HA (blank control), salivary pellicle-coated HA modified by heat-inactivated $2.5 \%$ CPP (negative control), or $2.5 \%$ CPP (CPP), or $2.5 \%$ CPP supplemented with 900 ppm fluoride (CPP + F). (a) Representative CLSM images at 30 min and $2 \mathrm{~h}$ of incubation. (b) Ratio (\%) of the area occupied by total fluorescence (total S. mutans) to the whole area. (c) Proportion (\%) 
of red fluorescence (dead S. mutans) to total fluorescence (total S. mutans). Data were expressed as the mean \pm SD. ${ }^{*} p<0.05,{ }^{*} p<0.01, * \star * p<0.001, * \star \star \star x p<0.0001$

Blank control Negative control $\quad$ CPP $\quad$ CPP + F
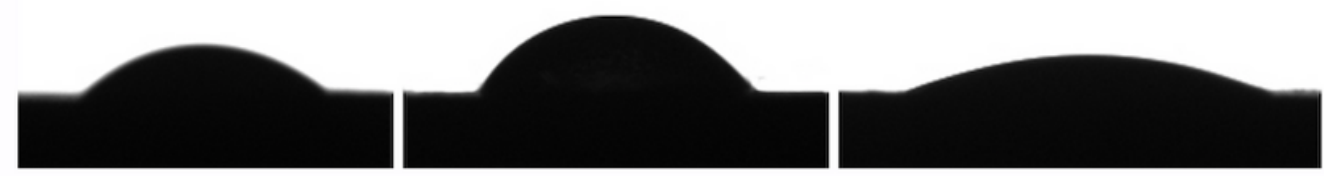

Figure 4

Representative images showing the contact angle of deionized water on the HA surface with different coatings: salivary pellicle-coated HA (blank control), salivary pellicle-coated HA modified by heatinactivated $2.5 \%$ CPP (negative control), or 2.5\% CPP (CPP), or 2.5\% CPP supplemented with $900 \mathrm{ppm}$ fluoride (fluoride-doped CPP). 